\title{
Fertility intention and related factors in China: findings from the 2017 National Fertility Survey
}

\author{
Yaer Zhuang ${ }^{1} \cdot$ Yu Jiang ${ }^{1} \cdot$ Bohua $\mathrm{Li}^{1}$
}

Received: 27 February 2020 / Accepted: 6 April 2020 / Published online: 20 April 2020

(C) The Author(s) 2020

\begin{abstract}
This paper reports the fertility intension of women based on data from the national fertility survey in 2017 in China (CFS 2017), after implementation of the universal two-child policy, and analyses major related factors. The reported ideal number of children is 1.96 in average, and the intended number of children is 1.76 , with obvious differences among different regions. The ideal number of children is highly correlated with the intended number of children, and intended number of children is related to number of children women already have. Women who have had one child intend to have fewer children than women who have not yet had a child. Women who have not yet had any child intend to have 1.60 children in average. The preference of boy varies by intended number of children.
\end{abstract}

Keywords Fertility intention · Ideal number of children · Intended number of children $\cdot$ Regional differences

\section{Introduction}

The relationships between fertility desire, fertility behavior and fertility level have been research focus of demographers for a long time. Because the fertility level of a population could be regarded as the aggregation of individual childbearing behavior, the relationship between fertility desire, fertility behavior, and fertility level is sometimes explained by "fertility desire and childbearing behavior", and sometimes expressed by "fertility desire and fertility level" in existing literatures. The difference is that the former emphasizes individual's opinion and behavior, while the latter is about a certain group or a population.

Since the 1970s, China has experienced a rapid decline in fertility, and it fell to the replacement level in the early 1990s and stayed low thereafter. According to the

Yu Jiang

jiangyu_cpdrc@126.com

1 China Population and Development Research Center, No. 12, Dahui Temple Road, Haidian District, Beijing city 100081, China 
results of the 2017 China Fertility Survey (hereinafter referred to as CFS 2017), during the period of 2006-2019, the annual average total fertility in China was 1.65, while 1.34 in cities and towns and 1.99 in rural areas, the difference between urban and rural areas is still significant (He et al. 2019). Although the national average has not yet dropped to a "very low" (below 1.5) level, the total fertility rates of cities and towns have approached 1.3, and that of rural areas have fallen below the replacement level of 2.1 .

In the context of low fertility, the most systematic expression of the relationship between fertility desire and fertility level is the theoretical framework by Bongaarts (2001) and the model developed from it (Morgan 2003). In this model, the total fertility rate, which represents the fertility level of a period, is affected not only by intended fertility, but also by multiple factors, namely, three positive factors (involuntary fertility, replacement effect, and gender preference) and three negative factors (tempo effect, infertility effect, and competition effect). According to the situation in China, some scholars estimated the parameters of the model and pointed out that the measurement of fertility desire in China would be affected by the fertility policy and the number of children a woman has. Due to the restrictions of the policy, the gender preference for boy is often realized through sex selective abortion, which makes the gender preference not a positive factor anymore (Guo 2008).

The rapid decline in China's fertility is under the dual influence of social economic development and family planning policy. The role of the family planning policy in the process of fertility decline could be divided into two categories, one is the direct restriction on childbearing behavior, and the other is that the relevant publicity and education has changed people's fertility desire so that childbearing behavior changed. The extraordinary decline in fertility in the 1970s and 1980s was not only due to the impact of mandatory factors, but also important voluntary factors (Chen 2005). In the current situation, with the successive implementation of reformed fertility policy to universal two-child policy, the direct restriction of the policy on childbearing behavior has been significantly weakened, and the impact of social and economic development on fertility desire and fertility level is becoming increasingly prominent.

There is no doubt that the study of fertility desire, as an important factor affecting childbearing behavior, is of great significance in the current situation. Based on the data of CFS 2017, this paper describes the fertility desire of Chinese women in the context of the universal two-child policy, and compares the differences between ideal number of children and fertility intention in different areas.

\section{Data and method}

The data used in this study come from a national fertility sampling survey, China Fertility Survey 2017 (CFS 2017), organized by National Health and Family Planning Commission in 2017. Women residents aged 15-60 who are currently living in the mainland were interviewed in July 2017. The survey adopts a stratified, three-stage and probabilities proportional to size sampling method. The sample is national representative. The questionnaire includes four parts: fertility desire, fertility behavior, childbearing 
and childrearing service use, and main factors affecting fertility status, in which the part of fertility behavior includes a complete history of pregnancy. Data quality issues have been discussed in terms of questionnaire and sample design, implementation process, quality control, data comparison, EX post facto weighting. Overall, the results of this survey are well representative of the country and the evaluation about the quality of the data is satisfactory (Zhuang et al. 2019).

Childbearing behavior has three dimensions: parity of child, timing of each child, gender of the child (Gu 1992). In the CFS 2017, the desire to have children is also measured by the above three dimensions, as the ideal number and ideal sex composition of children, the number and sex of children plan to have, and expected year to give birth to next child. We will introduce and analyze the survey results from two dimensions, that is, the ideal and intended number and sex of children. In CFS 2017, information about fertility desire and intention were collected among women aged 15-49 who are unmarried, or first-married, or remarried, or cohabiting, and there are 186,689 valid cases included in this study. The average ideal number of children and the average intended number of children obtained from the survey are not only well representative at national level, but also representative for each province, while for sex ratio is only representative at the national level due to the sample size limitation.

In the research on fertility desire, the ideal number of children is a frequently used indicator, which expresses the number of children without any conditions and constraints. In fact, no matter from the perspective of the family planning policy or socioeconomic context, it is very difficult in the actual situation to meet the requirement of "not being restricted by any conditions". Therefore, the ideal number of children obtained from some previous surveys may be under-estimated (Wang et al. 2013). Considering the impact of the fertility policy on the desire to have children, before the implementation of the universal two-child policy, the often asked question was a conditional one, such as "how many children do you plan to have if the policy allows?" We assume that the concern on policy restriction become less important after the implementation of the universal two-child policy in 2016. In CFS 2017, the questions about ideal number of children is "How many children do you think is ideal for a family?" and about intended number of children is "How many children do you plan to have?" We take the reported number of the first question as ideal number of children, and the reported number of second question (or plus number of children already have) as intended number of children. Note that the intended number of children consists of two parts: the number of children already have and the number of children plan to have in the future, so for those who have been married or in cohabitation, we combined results of children already have and children plan to have in the future as the intended number of children, or, number of children the women plan to have.

\section{Major findings}

\subsection{Ideal number of children and sex composition}

Information about ideal number of children was collected in four national surveys conducted in 2001 (by National Family Planning Commission), 2006 (by 
National Population and Family Planning Commission), 2013 (by National Health and Family Planning Commission), and 2017 (CFS 2017). The results are comparable after controlling the sample coverage, marital status (only first marriage and remarriage women included) and age range (20-44 years old). The ideal number of children in average is in a trend of gradually increasing: 1.72 in 2001 $(N=26,481), 1.76$ in $2006(N=22,079), 1.92$ in $2013(N=33,350)$, and 1.98 in $2017(N=101,255)$. It can be seen that the difference between 2001 and 2006 (implementation of the original family planning policy) as well as the difference between 2013 and 2017(improving and reforming the family planning policy) is small, while there is a "jump" from 2006 to 2013. Prior to the 2013 National Fertility Desire Survey, research activities on fertility policy reform had been carried out, in particular, the decision to "safely carry out pilot work on the policy that families with only one child can have a second child" was clearly mentioned in the draft document of "12th 5-year Plan for National population Development" distributed by the former National Population and Family Planning Commission in January 2010. These activities and messages may have an impact on the public opinion and could partly explain the significant gap between 2006 and 2013 survey results. Under the background of the increasing expectations of the public to adjust and improve the fertility policy, it may leads to an increase in reported ideal number of children.

The reported average ideal number of children is 1.96 in 2017. There are significant differences in the ideal number of children among some sub-populations, especially in different regions. The average ideal number of children by some groups is shown in Table 1. The increase in the average ideal number of children is associated with age, ranges from 1.85 for the youngest age group to 2.06 for the oldest age group. The average ideal number of children of women with agricultural hukou is larger than that of non-agricultural hukou, and sub-group of highest education has the lowest average ideal number of children among groups by education level. The average ideal number of children of women who have siblings is larger than that of women who are the only-child in family. The number of children ever born is highly related to the ideal number of children. This positive relationship between ideals and the number of children ever born shows a tendency that people think their existing children is close to ideal (Zhou and Huang 2000).

In each of the above-mentioned four national surveys, information was collected about ideal gender composition of children, as ideal number of boys and girls. Considering that "it doesn't matter boys and girls" happens randomly, such women were excluded when the sex ratio of the ideal number of children was calculated, then we estimated the sex ratio of the ideal children for married women of childbearing age in 4 years, it is 105.1 in 2001 survey $(N=33,402), 103.1$ in 2006 survey $(N=N=29,661), 101.0$ in 2013 survey $(\mathrm{N}=53,331)$, and 94.6 in 2017 survey $(N=199,962)$, showing a gradual downward trend. A study using meta-analysis from results of 152 surveys since 1979 finds that the number of ideal boys declined faster than the ideal number of girls in 2010-2017, as compared with the changes in 1979-2009, and led to a decline in the sex ratio of ideal children (Hou et al. 2018). The change of the sex ratio of ideal children in the four surveys is basically consistent with the findings by Hou et al. 


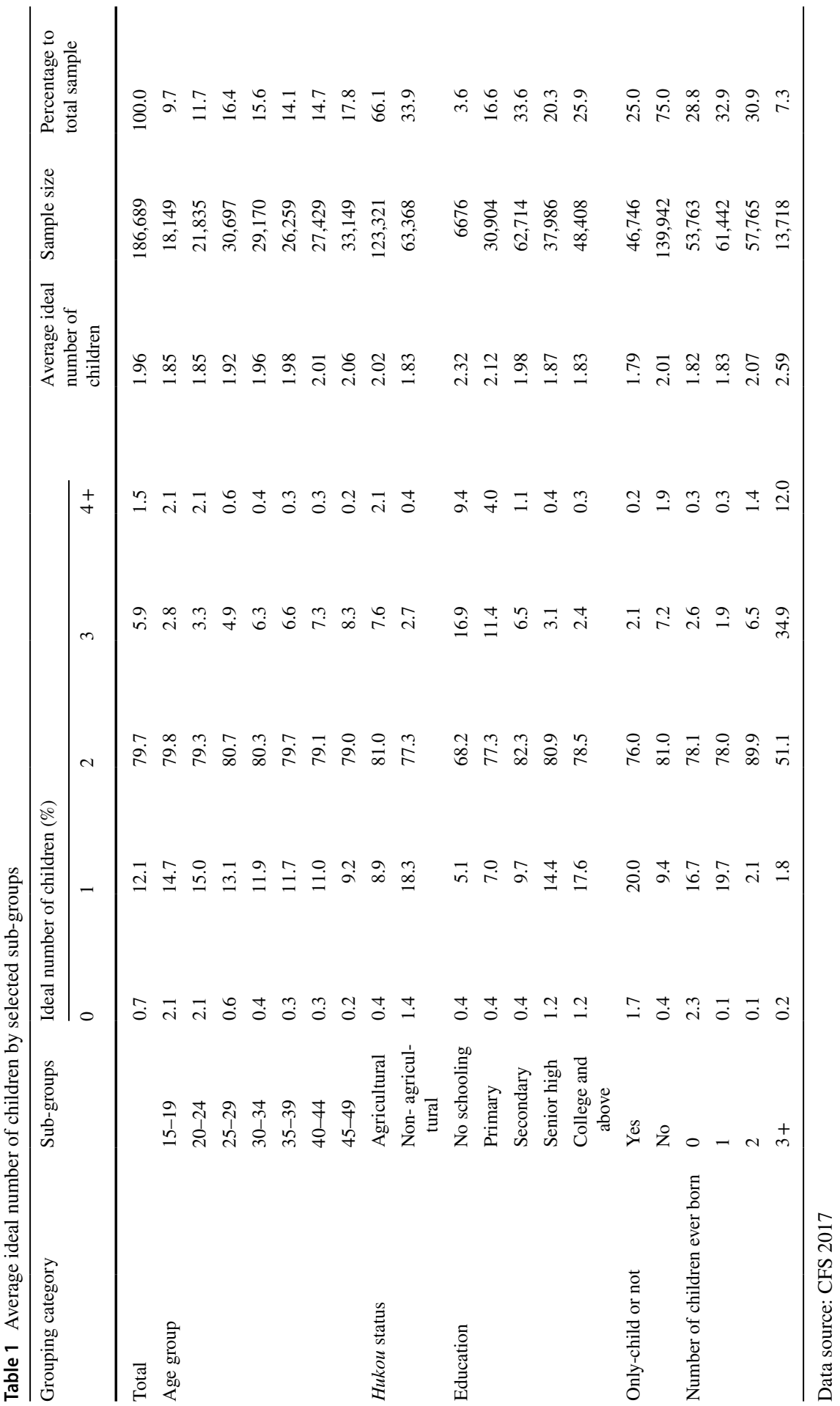


In the 2017 survey, the sex ratio of ideal children for both unmarried and married women of childbearing age $15-49$ is $93.5(N=354,287)$, in which the sex ratios of one child, two children, three children and more are $76.2(N=21,110), 94.8$ $(N=289,121)$, and $94.2(N=44,056)$, respectively.

When the ideal number of children is 1 child, the corresponding sex ratio is significantly lower. The gradual decline in the sex ratio of ideal children may be related to multiple factors such as the increasing competitive pressure of men in the marriage market in recent years and the fact that they do not necessarily have obvious advantages in supporting the elderly. The sex ratio at birth in China might to continue a slow downward trend in the future given the changing trend of gender preference.

\subsection{Intended number of children and sex composition}

The number of children a woman plan to have is the key measurement of intended number of children. If the ideal number of children is an idealized or nearly idealized norm, which more reflects people's opinion in the social and economic context, then the number of children one plan to have will be affected by the socioeconomic factors, and be more subject to the realistic conditions of the individual and the family. The intended number of children will be closer to the actual number of children than the ideal number of children.

The intended number of children of women in childbearing age is 1.76 . Table 2 shows there are three variables related to intended number of children, namely, the household registration (hukou) status, the level of education and whether the woman is the only child or not. The relationship of the three variables to the number of intended number of children is similar to that on the ideal number of children. The relationship between intended number of children and other two variables, age and the number of children ever born, is significantly different from the relationship with ideal number of children.

When grouped by age, the ideal number of children is associated with age, while the intended number of children shows a different pattern. The intended number of children increases with age at younger age groups, and reaches the peak of 1.83 at the age group 30-34, and then decreases afterwards. The pattern implies that young women in the peak of fecundity are more likely to have fewer children, and women in the middle age groups are not only intended but also capable to have another child, while among the older age groups there are combined effects of increased reproductive health risk by age, decreased fecundity and availability of childcare help from grandparents among women aged 35 and above.

The relationship between the number of children ever born and intended number of children is more complex than that relationship between number of children ever born and ideal number of children. The ideal number of children is positively related to the number of children ever born. However, Table 2 shows that the average intended number of children reported by women who have given birth to one child is significantly lower than that of women who have not yet had children (1.29 versus 1.60). This might be the result of multi-effects, including age and family 


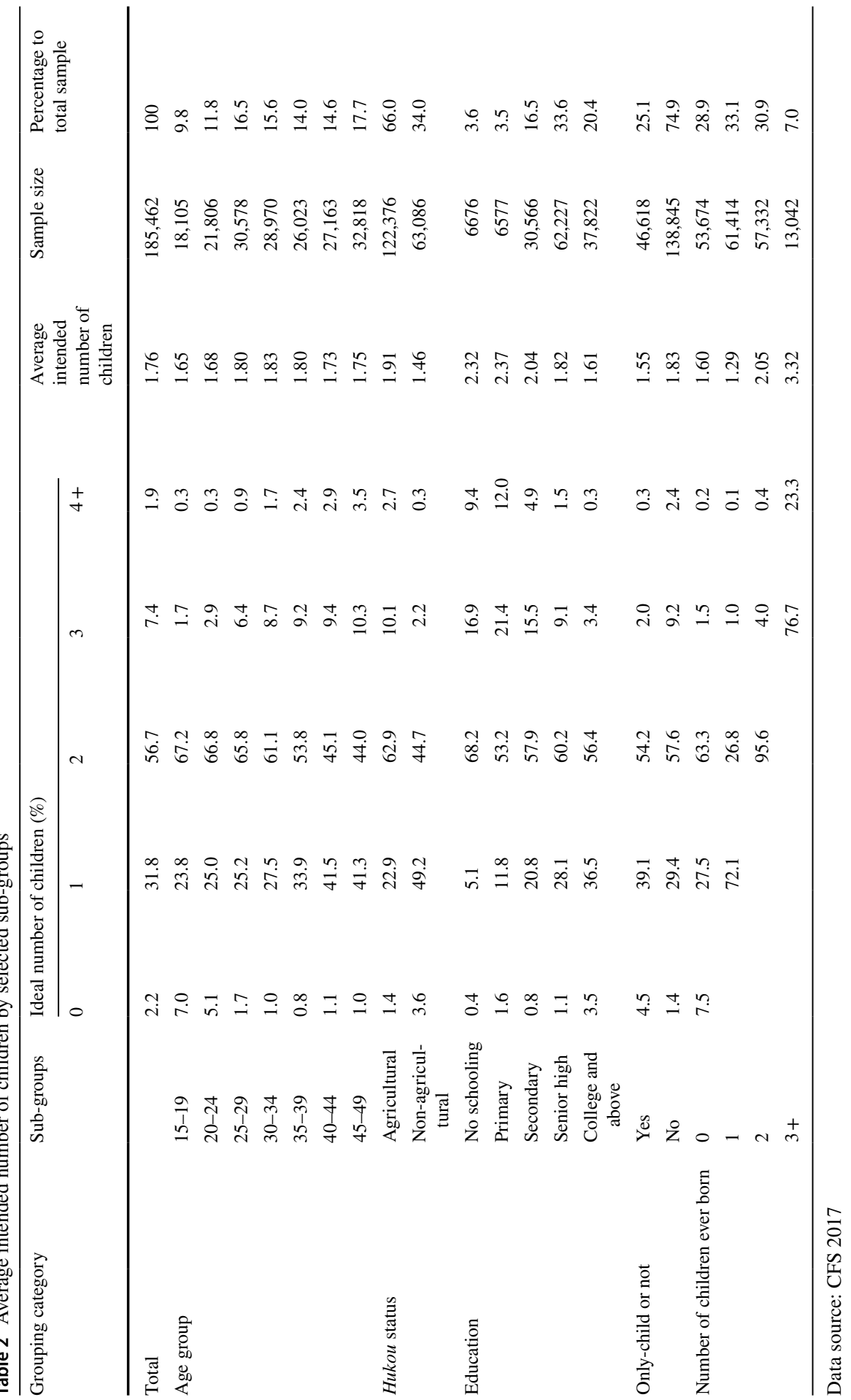


planning policy factors. For the four subgroups of the number of children ever born, the average age and the proportions of non-agricultural hukou of women with 0 child were 23.0 years old and $43.0 \%$, and the corresponding indicator for women with 1 child were 37.5 years old and $48.6 \%$, for women with 2 children were 38.5 years old and $16.5 \%$, and for women with 3 children and above were 40.5 years old and $6.6 \%$ respectively. As the average age of women who have given birth to one child reaches 37.5 years, such an age would have a negative impact on women plan to have another child. Furthermore, the proportion of non-agricultural hukou status is the highest in the one child group among the four sub-groups, probably due to the former family planning policy in cities (mainly one child per couple), which not only restricted urban couples' reproductive behavior, but also changed their childbearing opinion to a considerable extent, so that the indented number of children of one-child sub-group is significantly lower than other sub-groups. Note that the women who have not yet given birth at present will be the major childbearing population in the future, and the number of children that these women plan to have will determine the future fertility level of the country. With the influence of social and economic factors, marriage rate, divorce rate, and infertility rate, the actual number of children born would be less than that of intended number of children. As the average intended number of children of women not yet have any child is 1.60 , which is less than the 1.76 of national average, it seems that there is still room for the fertility rate to decline in the future.

The survey results show that the intended sex ratio of unmarried and married women of childbearing age is $103.8(N=316,398)$, and the intended sex ratio of one child, two children, three children and more is $123.1(N=57,157), 105.6$ $(N=203,964)$, and $81.7(N=55,277)$, respectively. Although on the whole, the overall average intended sex ratio of children is almost in balance, the sex ratio varies greatly with the number of children intended to have.

Among the women who plan to have one child, $22.5 \%$ have not yet given birth, and $77.5 \%$ have given birth to a child. The sex ratio corresponding to those who plan to have one child is as high as 123.1, mainly related to the sex of the child already born. Some scholars have commented that "if people only care about the sex of their children rather than the number of children, and do not want to have more children, they would have as few children as possible and effectively give birth to children of the ideal sex composition" (Qiao 2006). The term "effective" here refers to the phenomenon that some people achieve both quantitative and gender satisfaction through sex-selective abortion due to fertility squeeze and boy preference, which in fact limited number of birth.

The sex ratio for respondents intend to have two children is quite balanced because nearly $80 \%$ of them intend to have a boy and a girl. The intended sex ratio of three children or more is significantly lower. The number of women intended to have three children is nearly four times that of women intended to have three or more children, therefore the sex ratio intended three or more children is basically dominated by the intended sex ratio of three children.

Table 3 shows that among the 9997 women who intended and already have three children, the proportions of the first and second children are boys, one boy and one girl, and two girls are $16.0 \%(N=1597), 37.2 \%(N=3715)$, and $46.9 \%(N=4685)$, respectively. The number of women whose first and second children are both girls 
is about three times that of women have both boys for the first and second children, implies that women who have not yet given birth to boy would be more likely to have another child. Boy preference plays a role in increasing the number of births in this case. Although the sex ratio of the third child is significantly higher to this group, the sex ratio of the first and second children is only $52.8(N=19,994)$, so the overall intended sex ratio of three children is only 84.5 .

\subsection{Ideal and intended number of children by province}

There has been always a significant regional difference in fertility desire in China. Zhuang et al. (2014) described the regional differences in the ideal number of children before the fertility policy change took place after 2013. According to the results of the CFS 2017, Fig. 1 shows the distribution of average ideal number of children and average intended number of children by province. Correlation analysis shows that the average ideal number of children at provincial level has a significant negative correlation with per capita GDP in 2016 (the correlation coefficient is -0.486 , statistically significant at 0.01 ). In order to understand the relationship between the fertility desire and intention at the provincial level and the level of economic development, the figure also includes per capita GDP indicators at provincial level in 2016. The correlation pattern in Fig. 1 indicating that in general, the average ideal number of children is lower in economically developed areas, as well as the average intended number of children. However, exceptions and more special cases also exist.

Figure 2 is a visual display of the average intended number of children by province. The variance of the ideal number of children in 31 provinces is only 0.03 , while the variance of the intended number of children is 0.07 , indicating that compared with intended numbers of children, the ideal number of children in different regions are more "convergent". Correlation analysis shows that, similar to the ideal number of children, the average intended number of children in 31 provinces is negatively correlated with provincial GDP per capita in 2016 (statistically significant at 0.01 level, the correlation coefficient is - 0.586). In the existing analysis of fertility level, there is a phenomenon that the fertility rate is higher in areas with lower level of economic development and lower in areas with higher level of economic development. Figure 2 show that there is a similar situation between the intended number of children and the level of economic development, such as Guizhou, Guangxi and Yunnan with highest intended number of children, and Beijing, Tianjin and Shanghai are among the lowest intended number of children.

Note that the relationship between the level of economic development and intended number of children is not always follow the simple pattern mentioned above. Take Heilongjiang as an example, the average intended number of children (1.33) is the lowest in the country, while the province GDP per capita ranks 10th in the country (from low to high, the same below), which is in a lower layer of economic development. Some factors are believe to play roles in the low childbearing intention, such as the level of urbanization and industrialization has been higher in China in the history, and the proportion of non-agricultural population are relatively higher, the implementation of the family planning policy and its influence on 
people's childbearing opinion are relatively greater, therefore to have less children has become a norm in the area. Another factor is the current difficulties in the province's economic development, the GDP per capita ranking has dropped from 22nd in 1997 to 10th in 2016. Although the economic situation is still not bad, the economic downward trend is likely to increase pressure on couples who consider raising children, thus restraining the intended number of children.

\section{Conclusion and discussion}

After the implementation of the universal two-child policy, the ideal number of children among Chinese women has increased slightly. The ideal numbers of children for married women age 20-44 have been increased, observed from four surveys since 2001, and from about 1.72 in 2001 increased to 1.98 in 2017. In the national fertility survey in 2017, the reported ideal number of children is 1.96 in average, among women aged 15-49 who never married, first married, remarried, or in cohabitation, while the intended number of children is 1.76 .

The intended number of children by women who had given birth to one child is significantly lower, and the intended number of children by women who have not yet given birth is 1.60 , which is lower than the overall average. As the young women who have not yet have children will be the major childbearing group in the future, it seems that there is still room for the fertility rate in China to decline.

The distribution of the intended number of children in different areas is similar to that of the ideal number of children, but the ideal number of children is more convergent than the intended number of children, indicating that the fertility intention is restricted by more constrains. The difference in the intended number of children in different regions is not only related to the current level of social and economic development, but also to its historical evolution and changing trend. In this regard, Heilongjiang is quite typical.

The gradual decline in the sex ratio of ideal children may be related to the increasing competitive pressure of boys in the marriage market in recent years and the fact that they do not necessarily have obvious advantages in supporting the elderly. Furthermore, there are major factors promoting girl preference at the same time, such as significant increase of women in university and urbanization. Overall, the sex ratio of

Table 3 Distribution of women who intend to have and have already had three children by child order and sex combination (person $N=9997$ )

\begin{tabular}{lll}
\hline First child & Second child & Third child \\
\hline 3314 (male) & 1597 (male) & 698 (male) \\
& & 899 (female) \\
& 1717 (female) & 987 (male) \\
& & 730 (female) \\
6683 (female) & 1998 (male) & 1199 (male) \\
& & 799 (female) \\
& 4685 (female) & 743 (female) \\
& &
\end{tabular}

Data source: CFS 2017 


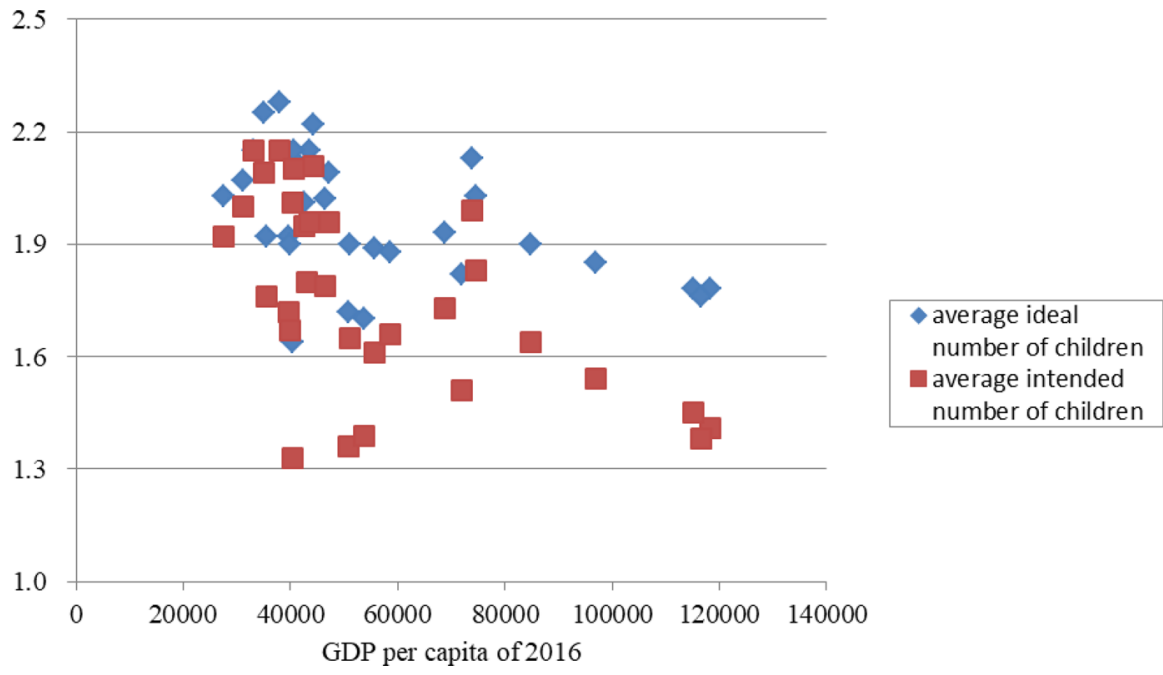

Fig. 1 Average ideal and intended number of children at province level by provincial GDP per capita. Data source: CFS 2017; China Statistical Yearbook 2017, National Bureau of Statistics of China, China Statistics Press, 2017

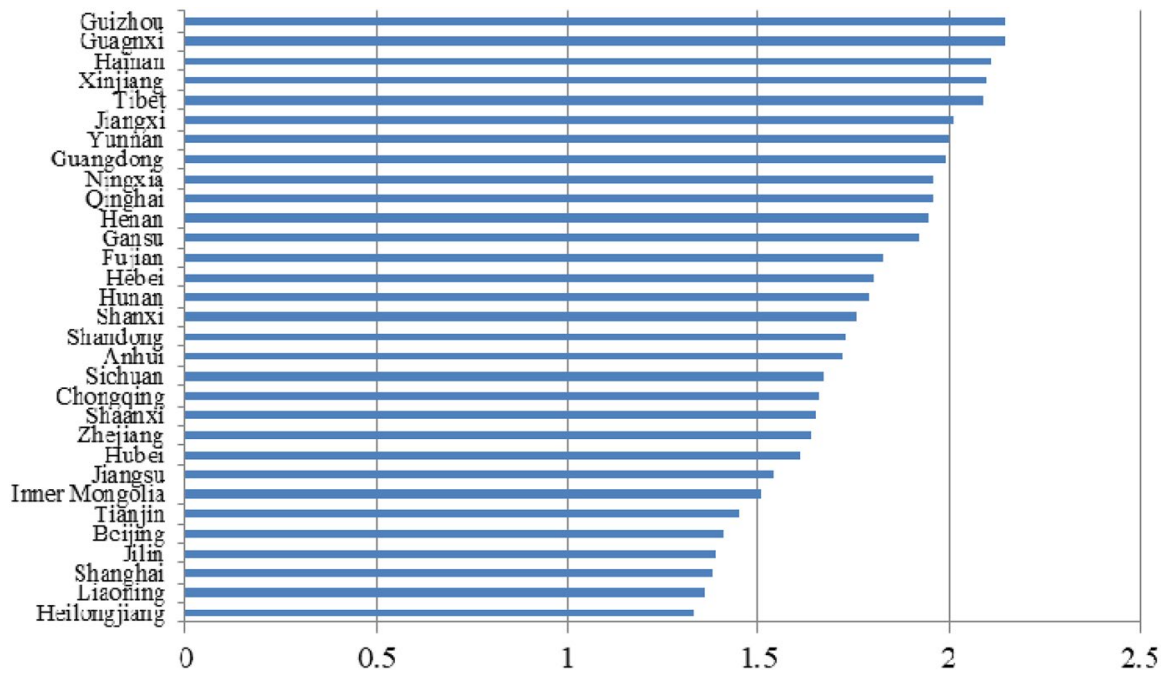

Fig. 2 Average intended number of children by province. Data source: CFS 2017

intended children is quite balanced; but due to boy preference, the intended sex ratio of one child is high, while the intended sex ratio of 3 children or more is on the low side. The influence of boy preference on fertility has two sides: on the one hand, boy preference and selective abortion limit and reduce the number of births; on the other hand, boy preference plays a role in increasing fertility without meeting the demand of "having at least one boy". 
Open Access This article is licensed under a Creative Commons Attribution 4.0 International License, which permits use, sharing, adaptation, distribution and reproduction in any medium or format, as long as you give appropriate credit to the original author(s) and the source, provide a link to the Creative Commons licence, and indicate if changes were made. The images or other third party material in this article are included in the article's Creative Commons licence, unless indicated otherwise in a credit line to the material. If material is not included in the article's Creative Commons licence and your intended use is not permitted by statutory regulation or exceeds the permitted use, you will need to obtain permission directly from the copyright holder. To view a copy of this licence, visit http://creativecommons.org/licen ses/by/4.0/.

\section{References}

Bongaarts, J. (2001). Fertility and Reproductive Preferences in Post-Transitional Societies. In R. A. Bulatao \& J. B. Casterline (Eds.), Global fertility transition (pp. 260-281). New York: Population Council.

Chen, W. (2005). Dynamic relationship between development, family planning and fertility rate in China: A reexamination using provincial level data. Population Research, 1, 2-10. (in Chinese).

Gu, B. (1992). On fertility and fertility transition: Quantity, time and sex. Population Research, 6, 35-45. (in Chinese).

Guo, Z. (2008). China's low fertility rate and its influencing factors. Population Research, 4, 1-12. (in Chinese).

Hou, J., Gu, B., \& Zhang, Y. (2018). The dynamic relationship between child preference and sex ratio at birth(1979-2017). Chinese Social Sciences, 10, 86-101. (in Chinese).

Morgan, S. P. (2003). Is low fertility a twenty-first-century demographic crisis? Demography, 40(4), $589-603$.

Qiao, X. (2006). A study of both theoretical and practical significance-a review of the article "fertility decline and gender imbalance under birth control". Market and Demographic Analysis, 4, 69-71. (in Chinese).

Wang, J., \& Wang, G. (2013). Fertility intention and its impact estimation of reproductive age population in China. Chinese Journal of Population Science, 4, 26-35. (in Chinese).

Zhou, C \& Huang, B. (2000). An analysis of the ideal fertility intention of women of childbearing age and its influencing factors. In Department of Planning and Finance of the State Family Planning Commission \& China Population Information Research Center (ed) Theses Collection of the 1997 National Population and Reproductive Health Survey (pp. 81-87). Beijing: China Population Publishing House. (in Chinese).

Zhuang, Y., Jiang, Y., Wang, Z., Li, C., Qi, J., Wang, H., et al. (2014). Current fertility intention of urban and rural residents in China: Based on the National Fertility Intention Survey in 2013. Population Research, 3, 3-13. (in Chinese).

Zhuang, Y., Yang, S., Qi, J., Li, B., Zhao, X., \& Wang, Z. (2019). China fertility survey 2017: Design and implementation. China Population and Development Studies, 2(3), 259-271.

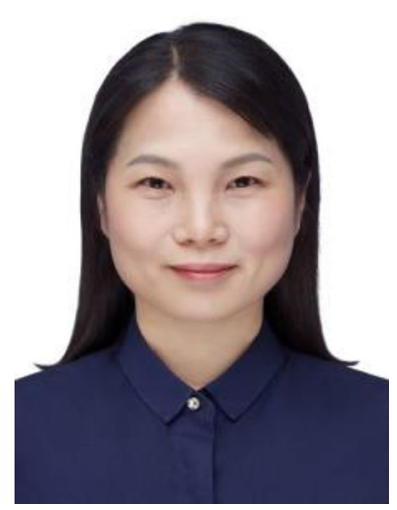

Yaer Zhuang is the deputy general secretary of China Population Association and the research fellow of China Population and Development Research Center, Beijing, 100081, China. 


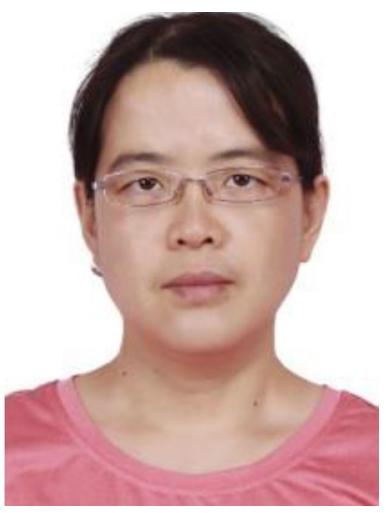

Yu Jiang is associate research fellow of China Population and Development Research Center, Beijing,100081, China.

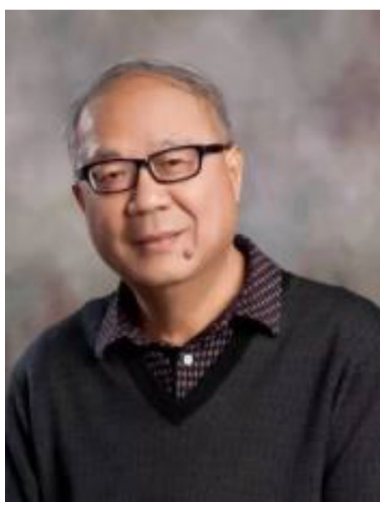

Bohua Li is associate research fellow of China Population and Development Research Center, Beijing,100081, China. 